\title{
Modeling Angiogenesis: A Discrete to Continuum Description
}

\author{
Samara Pillay, ${ }^{*}$ Helen M. Byrne, and Philip K. Maini \\ Wolfson Centre for Mathematical Biology, Mathematical Institute, \\ University of Oxford, Woodstock Road, Oxford, OX2 6GG, United Kingdom
}

(Dated: November 9, 2016)

\begin{abstract}
Angiogenesis is the process by which new blood vessels develop from existing vasculature. During angiogenesis, endothelial tip cells migrate via diffusion and chemotaxis, loops form via tip-to-tip and tip-to-sprout anastomosis, new tip cells are produced via branching and a vessel network forms as endothelial cells follow the paths of tip cells. The latter process is known as the snail-trail. We use a mean-field approximation to systematically derive a continuum model from a two-dimensional lattice-based cellular automaton model of angiogenesis in the corneal assay, based on the snail-trail process. From the two-dimensional continuum model, we derive a one-dimensional model which represents angiogenesis in two dimensions. By comparing the discrete and one-dimensional continuum models, we determine how individual cell behavior manifests at the macro-scale. In contrast to the phenomenological continuum models in the literature, we find that endothelial cell creation due to tip cell movement (vessel formation via the snail-trail) manifests as a source term of tip cells on the macro-scale. Further, we find that phenomenological continuum models, which assume that endothelial cell creation is proportional to the flux of tip cells in the direction of increasing chemoattractant concentration, qualitatively capture vessel formation in two dimensions, but must be modified to accurately represent vessel formation. Additionally, we find that anastomosis imposes restrictions on cell density, which, if violated, leads to ill-posedness in our continuum model. We also deduce that self-loops should be excluded when tip-to-sprout anastomosis is active in the discrete model to ensure propagation of the vascular front.
\end{abstract}

PACS numbers: 87.10.Ed, 87.10.Hk, 87.18.Gh

\section{INTRODUCTION}

Angiogenesis is the process by which new blood vessels develop from existing vasculature. Angiogenesis is important for wound healing, in developmental processes, such as embryogenesis, and in pathological conditions, such as cancer. Solid tumors initiate angiogenesis, a hallmark of cancer $[1,2]$, to increase nutrient and oxygen supply, and remove metabolic waste [2]. Typically, angiogenesis is initiated when hypoxic tumor cells secrete tumor angiogenic factors (TAFs) such as vascular endothelial growth factor (VEGF) [3-5]. TAFs diffuse towards the nearby vasculature, establishing a spatial gradient between the tumor and the vasculature. TAFs that reach the vasculature induce endothelial cells (ECs) lining blood vessels to degrade their basement membranes $[3,5,6]$ and migrate, via chemotaxis, up the spatial gradient of TAFs and towards the tumor [7], using filopodia to sense environmental guidance cues $[5,6,8]$ (see Fig. 1). ECs, or stalk cells $[5,6]$, follow the paths of leading ECs, known as tip cells (TCs). The ECs proliferate and elongate to form a snail-trail of capillary sprouts behind the leading TCs $[3,5,6]$.

As the sprouts approach the tumor, they fuse and form interconnected loops through tip-to-sprout and tip-to-tip anastomosis [5, 6] (see Fig. 1). Capillary loop formation and perfusion are essential for blood circulation and nu-

* samara.pillay@maths.ox.ac.uk trient supply. As the sprouts migrate towards the tumor, new TCs emerge through branching (see Fig. 1), a process which is stimulated by the TAFs [5]. Experimental results [9] in the corneal assay indicate that the density of the vascular network, specifically the density of TCs, increases as the tumor is approached, producing the socalled brush-border effect. Once the sprouts reach the tumor, vascularization occurs followed by reorganization and remodeling of the networks.

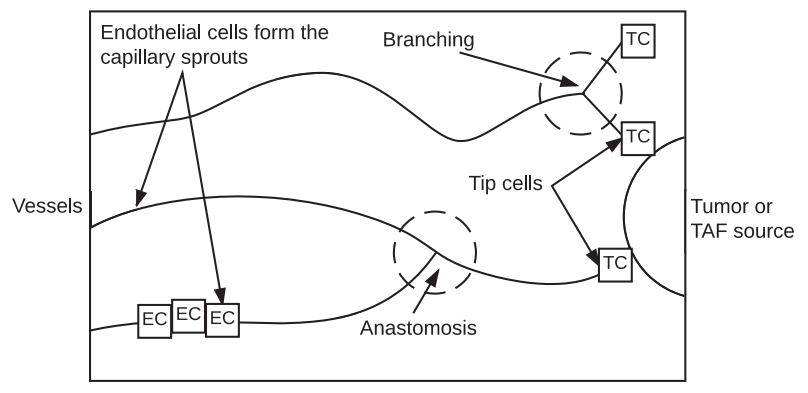

FIG. 1. A schematic of angiogenesis. Endothelial cells follow the paths of tip cells, which move chemotactically in response to a tumor angiogenic factor (TAF) source to form sprouts. Sprouts may branch and fuse through anastomosis to form loops. $\mathrm{EC}=$ endothelial cell, $\mathrm{TC}=$ tip cell.

Since angiogenesis is important for tumor growth and spread, the treatment of cancer via anti-angiogenic therapies is an active area of research [5, 10-12]. Paradoxically, certain anti-angiogenic treatments have been found 
to transiently "normalize" tumor vasculature, resulting in improved nutrient and drug supply [10]. Combination therapies, which administer anti-cancer drugs in conjunction with anti-angiogenic drugs, are a promising methodology for treatment [10-12]. Thus, for anti-angiogenic therapies to improve the prognosis of cancer patients, a more complete understanding of angiogenesis is necessary. To further elucidate the angiogenic process, an understanding of how cell-level (micro-scale) processes, such as vessel structure, affect tissue-scale (macro-scale) processes, such as nutrient and drug transport, is required. Given its importance in biology and the therapeutic drive for a more holistic understanding of angiogenesis, a large body of mathematical literature has been devoted to modeling angiogenesis (see the reviews Mantzaris et al. [13] and Scianna et al. [14] and references therein).

Mathematical models of angiogenesis can, broadly speaking, be split into two distinct types, namely discrete and continuum. Whilst discrete models can produce detailed vascular structures based on the micro-scale behavior of cells, continuum models, which describe the vessel network in terms of cell densities, provide a more tractable framework for analysis. Some of the earliest continuum models $[15,16]$, known as snail-trail models, were developed phenomenologically to establish qualitative agreement with angiogenesis in the corneal assay. Given its avascular nature, the corneal assay is widely used to study angiogenesis in vivo [17]. A TAF source implanted into the cornea of a small animal, usually a mouse [17], stimulates new vessels to emerge from the limbus, which forms the border between the cornea and sclera (the white of the eye), typically situated 1 to $2 \mathrm{~mm}$ from the TAF source $[9,18]$. Given the negligible thickness of the cornea (approximately $100 \mu \mathrm{m}$ in mice $[19,20]$ ), angiogenesis can be approximated as a two-dimensional process in the corneal assay. One-dimensional snail-trail models $[15,16]$ aim to capture two-dimensional processes by modeling the spatio-temporal evolution of vessel and TC densities, averaged in the direction perpendicular to propagation of the vascular front. Extending the model of Balding and McElwain [15], Byrne and Chaplain (BC) [16] assumed that the TCs move randomly and chemotactically in response to a generic diffusible TAF produced by a pellet or tumor fragment implanted into the cornea. The vessel network forms in response to TC movement such that the spatio-temporal evolution of the vessel density is proportional to the flux of TCs. Numerous other authors [21-24] have extended these snailtrail models $[15,16]$. Whilst one-dimensional snail-trail models are tractable, they describe physical quantities in terms of densities, and therefore do not represent the network morphology. Discrete models [25, 26] can account for the network structure, including the micro-scale behavior of cells. Anderson and Chaplain [27] and Chaplain [28] developed an agent-based model by discretizing a continuum model to define transition probabilities for the discrete model. Bentley et al. [29, 30] used an agent-based approach to model the inter- and intra-cell signaling mechanisms which mediate TC selection and migration. Hybrid models [31-34] couple discrete and continuum models; they model micro-scale cell behavior in response to macro-scale fields, such as TAFs, which are described by partial differential equations (PDEs). Hybrid models can also couple cell dynamics to models of blood flow [35-37] and drug delivery in the vascular network [35] as well as tumor growth [37]. These frameworks have been used to test the impact of different therapeutic strategies $[31,35]$.

As more experimental data become available, model validation becomes feasible. Tong and Yuan [33] and Connor et al. [24] have analyzed images of corneal angiogenesis to validate and parametrize their models. Experimental data may describe both cell- and population-level behavior. Thus, having continuum and discrete models can facilitate model validation at both scales, and taken together, provide complementary perspectives of angiogenesis. Increasing recognition of these benefits has stimulated efforts to connect discrete and continuum models in a variety of biological contexts [38-45], see the review by Codling et al. [46]. Typically, a lattice-based approach and a mean-field approximation, in which the occupancy of lattice sites is assumed to be independent, are used to derive continuum approximations to discrete models, though corrections to mean-field models have been investigated [47-49]. In the context of angiogenesis, Spill et al. [50] used a one-dimensional meso-scale lattice-based model to derive macroscopic descriptions of angiogenesis based on the snail-trail approach. In so doing, Spill et al. [50] reproduced the model developed by Byrne and Chaplain [16], introducing a norm to ensure the positivity of the TC flux. Bonilla et al. [51] developed a stochastic meso-scale model of TC dynamics, coupled to underlying macro-scale fields, and derived a deterministic integro-PDE system for the formation of the network using a mean-field approximation.

In this work, we determine the macroscopic behavior of TCs and ECs from their micro-scale behavior during angiogenesis. We develop a two-dimensional lattice-based cellular automaton (CA) model of angiogenesis in the corneal assay, based on the snail-trail process, and using a mean-field approximation, we systematically derive a new continuum model describing the spatio-temporal evolution of the TC and EC densities. From the twodimensional model, we derive a one-dimensional continuum model that can be used to represent angiogenesis in two dimensions. By comparing our discrete and onedimensional continuum models, we generate new biological insight by determining how individual cell behavior manifests itself at the macro-scale. This also enables us to elucidate, at a more fundamental level, the assumptions underlying phenomenological one-dimensional snail-trail models $[15,16]$.

In contrast to other snail-trail models [15, 16, 50], our systematic modeling approach reveals that anastomosis imposes restrictions on cell density in the continuum 
model. We also find that ECs created in response to TC movement on the micro-scale can be modeled as a TC source term on the macro-scale. Further, we find that phenomenological snail-trail models $[15,16]$ qualitatively capture the EC density (vessel formation) in two dimensions. In particular, these models only accurately capture vessel formation if TCs are restricted to motion in the direction of increasing TAF gradient and must be modified to account for TC movement and consequent EC creation in other directions. We have also deduced that for our CA model to produce a vascular front that propagates towards the TAF source, self-loops (a TC anastomoses with an EC from its own sprout) should be excluded when tipto-sprout anastomosis is active.

\section{CELLULAR AUTOMATON}

We represent the cornea as a two-dimensional, regular lattice, $(x, y) \in[0,1] \times[0,1]$, with lattice spacing $h$. Each lattice site is indexed by $(i, j)$ where $i, j, \in \mathbb{Z}^{+}$ $(0 \leq i, j \leq R, R h=1)$, so that the position of a site is given by $\left(x_{i}, y_{i}\right)=(i h, j h)$. The lattice spacing $h$ represents the diameter of a cell. The limbus and TAF source are located at $x=0$ and $x=1$, respectively.

Our CA model is presented in non-dimensionalized units, where we have rescaled distance with the limbus to TAF source separation $L$ and time with $L^{2} / D_{T A F}$ ( $D_{T A F}$ is the TAF diffusion coefficient). Generally, the TAF concentration (non-dimensional), $c(x, y, t)$, satisfies a reaction-diffusion equation $[16,24,27,52]$. Since the timescale for diffusion of TAFs is typically much faster than the timescale for changes in the vessel network (see [16]), we apply a quasi-steady-state approximation, (setting $\partial c / \partial t=0)$ and, for simplicity, assume that the TAF field is diffusion-dominated. Thus, the TAF field satisfies

$$
\begin{aligned}
& \nabla^{2} c=0, \text { for } 0 \leq x, y, \leq 1, \\
& c(0, y)=0, c(1, y)=1, \text { for } 0 \leq y \leq 1 \\
& \frac{\partial c}{\partial y}(x, 0)=0, \frac{\partial c}{\partial y}(x, 1)=0, \text { for } 0 \leq x \leq 1 .
\end{aligned}
$$

We have also assumed that TAFs are produced at a constant rate at $x=1$, and are removed by a sink at the limbus $(x=0)$. The solution to equations (1)-(3) is

$$
c(x, y)=x .
$$

This simplified TAF profile enables us to focus on the dynamics of TC movement and sprout creation.

Based on the biology of angiogenesis (TCs migrate and ECs follow the TCs), we distinguish two agent types in our lattice-based CA model; active TCs and passive ECs. TC dynamics are governed by the following processes: movement via random motion and chemotaxis towards the TAF source (at $x=1$ ), new TC formation through branching, and TC annihilation through tip-to-tip and tip-to-sprout anastomosis. ECs are created as a result of
TC movement (snail-trail process) and tip-to-tip anastomosis.

Our algorithm for updating TCs on the lattice is both random and sequential. If there are $\bar{N}$ TCs on the lattice at the beginning of a discrete time step $\bar{K}$, then $\bar{N}$ TCs are selected independently at random, with replacement. Once chosen, a TC is given the opportunity to move with probability, $P_{m}$. A further set of probabilities $P_{x \pm}$ and $P_{y \pm}$ dictate to which site a TC moves (described later). Once $\bar{N}$ motility attempts have been made, another $\bar{N}$ TCs are selected at random with replacement during the same discrete time step, and are given the opportunity to branch with probability $P_{b}$. ECs are not actively updated in the CA model, rather, they are created as a consequence of TC movement. We now discuss the specific rules governing TC motility and branching.

\section{A. Movement and Anastomosis}

If a TC at site $(i, j)$ within the lattice is given the opportunity to move, the probabilities $P_{x \pm}(i, j)$ and $P_{y \pm}(i, j)$, defined as

$$
\begin{aligned}
& P_{x \pm}(i, j)=\frac{1 \pm g_{x}(i, j)}{4}, 0<i, j,<R, \\
& P_{y \pm}(i, j)=\frac{1 \pm g_{y}(i, j)}{4}, 0<i, j,<R
\end{aligned}
$$

govern its ability to move from site $(i, j)$ to a site within its von Neumann neighborhood $(i \pm 1, j \pm 1)$. The functions $g_{x}$ and $g_{y}$, which bias the TC random walk, are central difference approximations to the TAF gradient at the location of the TC in the $x$ - and $y$-direction, respectively, and are assumed to represent the TC's ability to migrate up the TAF gradient via chemotaxis. We define $g_{x}$ and $g_{y}$ as follows

$$
\begin{aligned}
& g_{x}(i, j)=k\left(c_{i+1, j}-c_{i-1, j}\right), 0<i, j<R, \\
& g_{y}(i, j)=k\left(c_{i, j+1}-c_{i, j-1}\right), 0<i, j<R,
\end{aligned}
$$

where $c_{i, j}$ is the TAF concentration and the constant parameter $k$ (independent of time and space) scales the TAF gradient and therefore the chemotactic response, and is chosen so that $g_{x}, g_{y} \in[-1,1]$ to ensure that $P_{x \pm}, P_{y \pm} \in[0,1]$. For a linear TAF (equation (4)), $c_{i, j}=c_{i}, g_{y}=0$ and therefore $P_{y \pm}=\frac{1}{4}$. Our CA model is valid up until the first TC reaches the TAF source at $i=R, 0 \leq j \leq R$, and we do not allow TCs to cross the remaining lattice boundaries (no flux).

When a TC moves to a site within its neighborhood, an EC is left behind, creating the trail of vessels associated with the snail-trail.

If a TC moves to a site that is occupied, an anastomosis event occurs. We assume that anastomosis annihilates TCs. We distinguish two types of anastomosis events, depending on the occupancy of the site to which the TC moved. If the target site is occupied by a TC, then two TCs anastomose, a tip-to-tip anastomosis event 

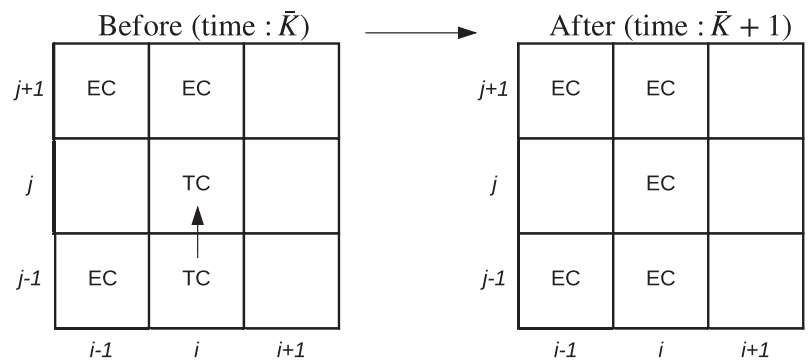

(a) Tip-to-tip anastomosis
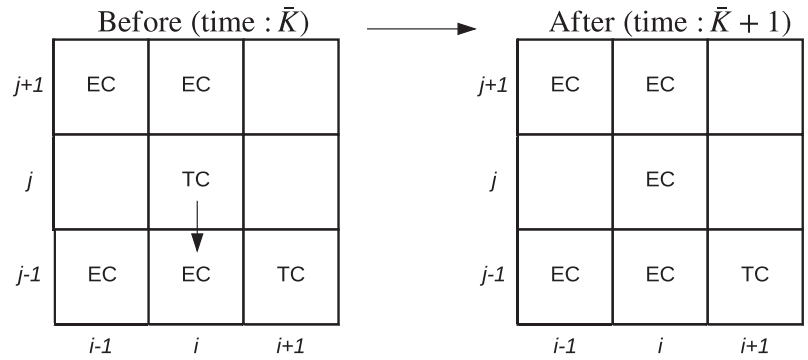

(b) Tip-to-sprout anastomosis

FIG. 2. (a) If a TC at site $(i, j-1)$ moves to site $(i, j)$, occupied by a TC, a tip-to-tip anastomosis event occurs. Both TCs are removed from the simulation and ECs are placed at site $(i, j)$ and $(i, j-1)$ due to anastomosis and movement, respectively. (b) If a TC at site $(i, j)$ moves to site $(i, j-1)$, occupied by an EC, a tip-to-sprout anastomosis event occurs. The TC is removed from the simulation, the EC at site $(i, j-1)$ remains and an EC is placed at site $(i, j)$ due to TC movement. $\mathrm{EC}=$ endothelial cell, $\mathrm{TC}=$ tip cell.

occurs, and an EC is deposited at the site of annihilation (i.e. TC + TC $\rightarrow$ EC; see Fig. 2(a)). If the target site is occupied by an EC, then a TC anastomoses with an EC, known as a tip-to-sprout anastomosis event, the TC is annihilated and the $\mathrm{EC}$ remains (i.e. $\mathrm{TC}+\mathrm{EC} \rightarrow \mathrm{EC}$; see Fig. 2(b)).

We introduce two binary variables, $a_{n}$ and $a_{e}$, to act as switches for tip-to-tip and tip-to-sprout anastomosis, respectively. If these variables are set to 1 , then the corresponding anastomosis process is active. In our CA model, we may allow movement only (i.e. no anastomosis, $\left.a_{n}=a_{e}=0\right)$, movement and tip-to-tip anastomosis $\left(a_{n}=1, a_{e}=0\right)$ or movement and tip-to-sprout anastomosis $\left(a_{n}=0, a_{e}=1\right)$, or movement and both tip-to-tip and tip-to-sprout anastomosis $\left(a_{n}=1, a_{e}=1\right)$.

\section{B. Branching}

We assume that branching occurs in the direction perpendicular to the direction of propagation of the TCs (towards the TAF source in the $x$-direction). Branching is stimulated by TAFs [5]. Therefore, we assume that the probability, $P_{b}$, with which a TC at site $(i, j)$ branches, increases linearly with the TAF concentration, $c$, such

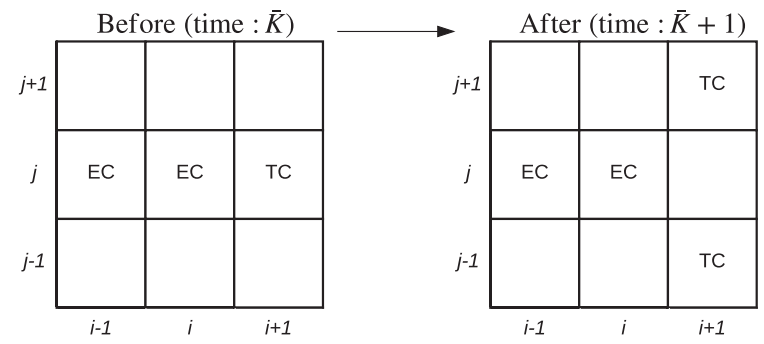

FIG. 3. A schematic showing how branching is accomplished in the CA model. A TC at site $(i+1, j)$ may branch during a time step by placing daughter TCs at $(i+1, j \pm 1)$. EC = endothelial cell, $\mathrm{TC}=$ tip cell.

that

$$
P_{b}(i, j)=P_{p} c_{i, j} \in[0,1],
$$

where $P_{p}$ is a constant (independent of time and space) that scales the TAF concentration. Branching from a TC located at $(i, j)$ places daughter TCs at $(i, j \pm 1)$ and the TC at site $(i, j)$ is removed (see Fig. 3). We assume that ECs are not created through branching. The resulting Y-pattern (see Fig. 3) simulates the branching pattern and TC selection that is in practice mediated by complex signaling involving the DLL4/Notch pathway $[5,6]$. Given that new TCs predominantly emanate from the front of the propagating vascular front (brush-border effect [9] observed in experiments), we assume branching occurs from the TCs only.

\section{Initial Conditions}

TCs are placed along $i=0$ at alternating lattice sites i.e.

$$
\text { TCs placed at }(i=0, j=1,3, \cdots, R-1) \text {, }
$$

representing sprouting from the corneal limbal vessels, following the initiation of angiogenesis. Initially, there are no ECs on the lattice. ECs are created when the TCs begin to move. In practice, as with branching, TC selection at the limbus is mediated by TAF (VEGF) and Notch signaling [5, 6, 53].

The CA algorithm for updating TCs after initialization is outlined in Appendix B.

\section{Cell Occupancy}

Branching and movement (incorporating anastomosis) have been implemented as two separate processes in our CA model. Each process affects the occupancies of the CA model differently.

Movement does not incorporate volume exclusion explicitly i.e. a TC move is not aborted if the target site is occupied. However, anastomosis does impose occupancy 
restrictions on the $\mathrm{CA}$. In particular, tip-to-tip anastomosis $\left(a_{n}=1, a_{e}=0\right)$ implies that only one TC can occupy a site (though a TC and an EC can occupy the same site), tip-to-sprout anastomosis $\left(a_{n}=0, a_{e}=1\right)$ implies that a TC and EC cannot occupy the same site (though multiple TCs can occupy the same site), and taken together, tip-to-tip and tip-to-sprout anastomosis $\left(a_{n}=1, a_{e}=1\right)$ imply that no two cells of any kind can occupy the same site.

Branching does not incorporate volume exclusion i.e. branching may deposit daughter TCs at sites already occupied by other TCs or ECs. Therefore, branching does not adhere to the occupancy restrictions imposed by anastomosis. We will discuss this issue further later on (see Section III B).

These rules may lead to cases where multiple TCs occupy a site (produced either by branching or tip-to-sprout anastomosis $\left.\left(a_{n}=0, a_{e}=1\right)\right)$ or cases where TCs occupy the same site as an EC (produced either by branching or tip-to-tip anastomosis $\left.\left(a_{n}=1, a_{e}=0\right)\right)$. As a result, multiple ECs can be deposited at that site as these TCs are updated and given the opportunity to move.

When branching and both anastomosis processes $\left(a_{n}=1, a_{e}=1\right)$ are implemented, we may encounter cases where a TC moves to a site that is occupied by both TCs and ECs (occurs through branching). We therefore impose the hierarchy that a tip-to-tip anastomosis event occurs first (see CA algorithm in Appendix B).

We also remark that though a target site may be occupied by multiple TCs and/or ECs, only one anastomosis event can occur during a TC move, and therefore involves only one cell at the target site (see Figs. 2(a) and 2(b)).

\section{E. Ensemble Averages}

To relate the discrete model to a continuum, macroscopic description, we average the occupancy of site $(i, j)$ over $M$ realizations of the discrete model at discrete times $1,2, \cdots, \bar{K}$. In particular, we define $n_{i, j}(\bar{K})$ and $e_{i, j}(\bar{K})$ as follows:

$$
n_{i, j}(\bar{K})=\frac{1}{M} \sum_{m=1}^{M} n_{i, j}^{m}(\bar{K}), \quad e_{i, j}(\bar{K})=\frac{1}{M} \sum_{m=1}^{M} e_{i, j}^{m}(\bar{K}),
$$

where $n_{i, j}^{m}(\bar{K})$ and $e_{i, j}^{m}(\bar{K})$ are the TC and EC occupancies of site $(i, j)$ after $\bar{K}$ discrete time steps at the $m$-th realization, respectively. We also define the column averages as follows:

$$
\begin{aligned}
& N_{i}(\bar{K})=\frac{1}{M R} \sum_{m=1}^{M} \sum_{j=1}^{R} n_{i, j}^{m}(\bar{K}), \\
& E_{i}(\bar{K})=\frac{1}{M R} \sum_{m=1}^{M} \sum_{j=1}^{R} e_{i, j}^{m}(\bar{K}),
\end{aligned}
$$

where $R$ is the number of columns on the lattice.

\section{THE CONTINUUM MODEL}

We derive our continuum PDE model by formulating discrete conservation equations (DCEs) that relate the average TC and EC occupancies at site $(i, j)$ at time $\bar{K}+$ $1, n_{i, j}(\bar{K}+1)$ and $e_{i, j}(\bar{K}+1)$ (ensemble averages defined in equation $(11))$, to the average occupancies at site $(i, j)$ at time $\bar{K}$ and the change in occupancy during a discrete time step. In formulating this conservation equation for the TCs and ECs, we must include TC movement due to diffusion and chemotaxis, anastomosis and branching, as well as the creation of ECs due to TC movement and tip-to-tip anastomosis. Defining $\delta n_{i, j}$ and $\delta e_{i, j}$ by

$$
\begin{aligned}
\delta n_{i, j} & =n_{i, j}(\bar{K}+1)-n_{i, j}(\bar{K}), \\
\delta e_{i, j} & =e_{i, j}(\bar{K}+1)-e_{i, j}(\bar{K}),
\end{aligned}
$$

the DCEs for the TC and EC population within the lattice $(0<i, j<R)$ are, respectively,

$$
\begin{aligned}
\delta n_{i, j}= & P_{m}(\underbrace{P_{x+}(i-1, j) n_{i-1, j}+P_{x-}(i+1, j) n_{i+1, j}+P_{y+}(i, j-1) n_{i, j-1}+P_{y-}(i, j+1) n_{i, j+1}}_{\text {movement into }(i, j)} \\
& -\underbrace{\left(P_{x+}(i, j)+P_{x-}(i, j)+P_{y+}(i, j)+P_{y-}(i, j)\right) n_{i, j}}_{\text {movement out of }(i, j)} \\
& -\underbrace{a_{n} n_{i, j}\left(P_{x+}(i-1, j) n_{i-1, j}+P_{x-}(i+1, j) n_{i+1, j}+P_{y+}(i, j-1) n_{i, j-1}+P_{y-}(i, j+1) n_{i, j+1}\right)}_{\text {tip-to-tip anastomosis }} \\
& -\underbrace{a_{e} e_{i, j}\left(P_{x+}(i-1, j) n_{i-1, j}+P_{x-}(i+1, j) n_{i+1, j}+P_{y+}(i, j-1) n_{i, j-1}+P_{y-}(i, j+1) n_{i, j+1}\right)}_{\text {tip-to-sprout anastomosis }}) \\
& +\underbrace{P_{p} c_{i, j}\left(n_{i, j-1}+n_{i, j+1}-n_{i, j}\right)}_{\text {branching }},{ }
\end{aligned}
$$


and

$$
\begin{aligned}
\delta e_{i, j}= & P_{m}(\underbrace{P_{x+}(i, j)+P_{x-}(i, j)+P_{y+}(i, j)+P_{y-}(i, j)}_{\text {movement of TCs out of }(i, j)} \\
& +\underbrace{a_{n}\left(P_{x+}(i-1, j) n_{i-1, j}+P_{x-}(i+1, j) n_{i+1, j}+P_{y+}(i, j-1) n_{i, j-1}+P_{y-}(i, j+1) n_{i, j+1}\right)}_{\text {tip-to-tip anastomosis }}) n_{i, j} .
\end{aligned}
$$

Equations (16) and (17) describe how TC and EC occupancy, respectively, change during a time step assuming that the occupancy of lattice sites is independent (meanfield approximation). We remark that equations (16) and (17) have been written in generality, and that for a linear TAF, $P_{y \pm}=\frac{1}{4}$.

The first line on the right-hand side of equation (16) represents TC movement from sites $(i \pm 1, j)$ and $(i, j \pm$ 1 ) into site $(i, j)$. TC movement from site $(i, j)$ into a neighboring site is represented by the second line. The third and fourth lines model tip-to-tip and tip-to-sprout anastomosis, respectively. If a TC from a neighboring site moves into site $(i, j)$ and it is already occupied by a TC or EC, then the TCs are lost, and a new EC is deposited at $(i, j)$ for tip-to-tip anastomosis, whilst for tip-to-sprout anastomosis, the EC at site $(i, j)$ remains. The fifth line on the right-hand side of equation (16) represents branching into the site $(i, j)$ from a TC located at $(i, j-1)$ or $(i, j+1)$ (source terms), and the loss of a TC from site $(i, j)$ if it branches into neighboring sites (sink term).

We can rewrite the first four lines of equation (16) governing TC movement and anastomosis as

$$
\begin{aligned}
& P_{m}\left(\left(P_{x+}(i-1, j) n_{i-1, j}+P_{x-}(i+1, j) n_{i+1, j}+P_{y+}(i, j-1) n_{i, j-1}+P_{y-}(i, j+1) n_{i, j+1}\right)\left(1-a_{n} n_{i, j}-a_{e} e_{i, j}\right)\right. \\
& \left.-\left(P_{x+}(i, j)+P_{x-}(i, j)+P_{y+}(i, j)+P_{y-}(i, j)\right) n_{i, j}\right) .
\end{aligned}
$$

Written in this form, we conclude that when anastomosis is active (i.e. $a_{n} \neq 0$ or $a_{e} \neq 0$ ) a TC can always move out of site $(i, j)$, with the outcome depending on the occupancy of the target site. A TC only remains a TC if the target site is vacant.

In equation (17), ECs are created when a TC moves out of a site and via tip-to-tip anastomosis. These source terms are the counterparts of sink terms in equation (16).
We now relate the DCEs to a continuum description. We expand all dependent variables, $n, e$ and $c$, in equations (16) and (17) in a Taylor series about the site $(i, j)$. Recall that $P_{x \pm}$ and $P_{y \pm}$ are defined in equations (5) and (6), respectively. By setting $x_{i} \rightarrow x, y_{j} \rightarrow y$, $n_{i, j}(\bar{K}) \rightarrow n(x, y, t), e_{i, j}(\bar{K}) \rightarrow e(x, y, t)$ and dividing the resulting expressions by $\tau$, we eventually arrive at the following expressions:

$$
\begin{aligned}
\frac{1}{\tau}(n(x, y, t+\tau)-n(x, y, t))= & \left(1-a_{n} n-a_{e} e\right) \frac{P_{m}}{\tau}\left(\frac{h^{2}}{4} \nabla^{2} n-h^{2} k \nabla \cdot(n \nabla c)+O\left(h^{4}\right)\right)-\frac{P_{m}}{\tau}\left(a_{n} n^{2}+a_{e} n e\right) \\
& +\frac{P_{p} c}{\tau}\left(n+h^{2} \frac{\partial^{2} n}{\partial y^{2}}+O\left(h^{4}\right)\right)
\end{aligned}
$$

and

$$
\frac{1}{\tau}(e(x, y, t+\tau)-e(x, y, t))=\frac{P_{m} n}{\tau}\left(1+a_{n} n\right)+\frac{a_{n} P_{m} n}{\tau}\left(\frac{h^{2}}{4} \nabla^{2} n-h^{2} k \nabla \cdot(n \nabla c)+O\left(h^{4}\right)\right),
$$

for the TC and EC populations, respectively.

By taking the limit as $\tau \rightarrow 0$, and neglecting terms of $O\left(h^{4}\right)$ and higher, we can relate our DCEs to a PDE model, and we find that $n(x, y, t)$ and $e(x, y, t)$ satisfy

$$
\begin{aligned}
\frac{\partial n}{\partial t}= & \left(1-a_{n} n-a_{e} e\right)\left(D \nabla^{2} n-\chi \nabla \cdot(n \nabla c)\right) \\
& -\mu\left(a_{n} n^{2}+a_{e} n e\right)+c\left(\lambda n+D_{b} \frac{\partial^{2} n}{\partial y^{2}}\right),
\end{aligned}
$$


and

$$
\frac{\partial e}{\partial t}=\mu n+a_{n} n\left(\mu n+D \nabla^{2} n-\chi \nabla \cdot(n \nabla c)\right)
$$

respectively, where

$$
\mu=\lim _{\tau \rightarrow 0} \frac{P_{m}}{\tau}, \quad \lambda=\lim _{\tau \rightarrow 0} \frac{P_{p}}{\tau},
$$

and the diffusion coefficient, $D$, chemotactic coefficient, $\chi$, and diffusion coefficient due to branching, $D_{b}$, are defined as

$$
\begin{aligned}
& D=\frac{\mu h^{2}}{4}=\lim _{\tau \rightarrow 0} \frac{P_{m} h^{2}}{4 \tau}, \quad \chi=\mu k h^{2}=\lim _{\tau \rightarrow 0} \frac{P_{m} k h^{2}}{\tau} \\
& D_{b}=\lambda h^{2}=\lim _{\tau \rightarrow 0} \frac{P_{p} h^{2}}{\tau} .
\end{aligned}
$$

These expressions for $D, \chi$ and $\lambda$ are consistent with existing literature $[39,40,46]$, though we have not taken the limit $\tau, h \rightarrow 0$ with $h^{2} / \tau$ constant. If we took the limit as $h \rightarrow 0$ with $\mu$ defined by equation (23), the diffusive and chemotactic terms, as well as the diffusive term due to branching, would vanish. Our PDE for $n$ would involve only source terms, which is at odds with our CA model, where TCs move by diffusion and chemotaxis. By defining $\bar{D}=\mu / 4, \bar{\chi}=\mu k$ and $\bar{D}_{b}=\lambda$, and rewriting $D$, $\chi$ and $D_{b}$ as $D=h^{2} \bar{D}, \chi=h^{2} \bar{\chi}$ and $D_{b}=h^{2} \bar{D}_{b}$, respectively, it is clear that in the limit as $h \rightarrow 0$, we have a singular perturbation problem. Since we use small nonzero values for $h$ and $\tau$ in the discrete simulations, and to define the continuum model parameters, we must retain the $O\left(h^{2}\right)$ terms in $(21)$ and (22) in order to capture the boundary layer in which the TC density changes rapidly. Similar approaches in which the limit $h \rightarrow 0$ was not explicitly taken or $O\left(h^{2}\right)$ diffusive terms were retained were used in $[43-45]$ to derive PDE approximations to discrete agent-based models of growing domains. We have neglected terms of $O\left(h^{4}\right)$ and higher in our model and we will show later that when these higher-order terms are neglected, the PDEs approximate the discrete model well.

We approximate the boundary conditions in the discrete model by imposing no flux boundary conditions on equations (21) and (22), assuming that anastomosis and branching are negligible there. With these boundary conditions, our PDE model remains valid until the TC front reaches $x=1$.

The initial condition for the TCs in the discrete model (see equation (10)) is discontinuous. We instead use the averaged CA simulation results for the TCs and ECs at some later discrete time step $\bar{K}_{I C}, n_{i, j}\left(\bar{K}_{I C}\right)$ and $e_{i, j}\left(\bar{K}_{I C}\right) \forall i, j$ (ensemble averages defined in equation (11)), respectively, as the initial conditions in the continuum model. Here, $t_{I C}$ is related to the discrete time step $K_{I C}$ by $t_{I C}=\tau \bar{K}_{I C}$.
Our continuum model, valid for $t \geq t_{I C}$, can now be written as:

$$
\begin{aligned}
\frac{\partial n}{\partial t}= & \left(1-a_{n} n-a_{e} e\right)\left(D \nabla^{2} n-\chi \nabla \cdot(n \nabla c)\right) \\
& -\mu\left(a_{n} n^{2}+a_{e} n e\right)+c\left(\lambda n+D_{b} \frac{\partial^{2} n}{\partial y^{2}}\right), \\
\frac{\partial e}{\partial t}= & \mu n+a_{n} n\left(\mu n+D \nabla^{2} n-\chi \nabla \cdot(n \nabla c)\right),
\end{aligned}
$$

subject to

$$
\begin{aligned}
& D \frac{\partial n}{\partial x}-\chi n \frac{\partial c}{\partial x}=0, \text { at } x=0,1,0 \leq y \leq 1 \\
& D \frac{\partial n}{\partial y}-\chi n \frac{\partial c}{\partial y}=0, \text { at } y=0,1,0 \leq x \leq 1
\end{aligned}
$$

with

$$
\begin{gathered}
n\left(x, y, t_{I C}\right) \forall x, y=n_{i, j}\left(\bar{K}_{I C}\right) \forall i, j, \\
e\left(x, y, t_{I C}\right) \forall x, y=e_{i, j}\left(\bar{K}_{I C}\right) \forall i, j .
\end{gathered}
$$

It is clear that terms multiplied by $D$ or $\chi$ only arise from the diffusive and chemotactic motion of the TCs, terms multiplied by $\lambda$ and $D_{b}$ arise from TC branching, and terms multiplied by $a_{n}$ or $a_{e}$ arise from tip-to-tip and tip-to-sprout anastomosis, respectively. The EC population is created predominantly through TC movement, via random motion and/or chemotaxis, as indicated by the source term $\mu$ on the right-hand side of equation (27). Here $\mu$ can be interpreted as the TC motility rate. We also note that given the form of equation (27), we need only specify an initial condition for $e(x, y, t)$. Equations (26)-(30) constitute a new (non-dimensional) model of angiogenesis. Whilst the terms that govern TC diffusion and chemotaxis are standard (see $[15,16,27,52]$ ), the terms associated with anastomosis, TC branching in two dimensions, and most notably, EC creation, are new.

We note that the diffusive term due to branching would read $D_{b} \nabla^{2} n$ had we implemented branching in the $x$ direction. Recall that we assumed that branching occurs only in the direction perpendicular to the direction of propagation of the vascular front (i.e. in $y$ only). We also assumed that branching does not create ECs, and therefore equation (27) does not contain source terms arising from branching. Our implementation of branching at the micro-scale gives rise to a branching term, $\lambda c n$, at the macroscale that is consistent with those used in existing continuum models $[16,50]$. We note that we could have implemented branching in many ways, as the leading order branching term, $\lambda c n$, describes a multitude of branching processes on the micro-scale (see Simpson et al. [39]). We also note that, for a linear TAF, equation $(4), \partial c / \partial y=0$ in the no flux boundary condition in $y$ (equation 29). 


\section{A. One-Dimensional Model}

We reduce our PDE model from two spatial dimensions to one by defining the following variables

$$
N(x, t)=\int_{0}^{1} n(x, y, t) d y, \quad E(x, t)=\int_{0}^{1} e(x, y, t) d y .
$$

Integrating equations (26) and (27) with respect to $y$, and using the definitions above, we find that $N(x, t)$ and $E(x, t)$, for $t \geq t_{I C}$, satisfy

$$
\begin{aligned}
\frac{\partial N}{\partial t}= & \left(1-a_{n} N-a_{e} E\right)\left(D \frac{\partial^{2} N}{\partial x^{2}}-\chi \frac{\partial}{\partial x}\left(N \frac{\partial c}{\partial x}\right)\right) \\
& -\mu\left(a_{n} N^{2}+a_{e} N E\right)+\lambda c N, \\
\frac{\partial E}{\partial t}= & \mu N+a_{n} N\left(\mu N+D \frac{\partial^{2} N}{\partial x^{2}}-\chi \frac{\partial}{\partial x}\left(N \frac{\partial c}{\partial x}\right)\right),
\end{aligned}
$$

subject to

$$
D \frac{\partial N}{\partial x}-\chi N \frac{\partial c}{\partial x}=0, \text { at } x=0,1
$$

with

$$
N\left(x, t_{I C}\right)=N_{i}\left(\bar{K}_{I C}\right), E\left(x, t_{I C}\right)=E_{i}\left(\bar{K}_{I C}\right), \forall x, i,
$$

where $N_{i}$ and $E_{i}$ are column ensemble averages as defined in equations (12) and (13), respectively. By reducing the model in this manner, we are making the following approximations

$$
\begin{aligned}
& \int_{0}^{1} n^{2} d y \approx\left(\int_{0}^{1} n(x, y, t) d y\right) \times\left(\int_{0}^{1} n(x, y, t) d y\right) \\
& \int_{0}^{1} n e d y \approx\left(\int_{0}^{1} n(x, y, t) d y\right) \times\left(\int_{0}^{1} e(x, y, t) d y\right) .
\end{aligned}
$$

We will show later that with these approximations, the PDEs, equations (31) and (32), approximate the discrete model well. We can also derive the one-dimensional equations directly from the DCEs, equations (16) and (17), by summing over $j$, and using the discrete analogue of the approximations above (mean-field approximation), which amounts to replacing the ensemble averages with their column averages (equivalent to replacing $n$ and $e$ with $N$ and $E$ in equations (26) and (27)). Equation (32) reveals that in the absence of tip-to-tip anastomosis $\left(a_{n}=0\right)$, the spatio-temporal evolution of the EC density is proportional to a source term of TCs, $\mu n$. In contrast, onedimensional phenomenological $[15,16]$ snail-trail models (see Appendix A) assume that EC (vessel) creation is proportional to the flux of TCs in the $x$-direction whilst Spill et al. [50] found that EC creation is proportional to a source term of TCs if TCs undergo diffusion only.

We compare the solutions of equations (31) and (32) to the column ensemble averages, defined in equations (12) and (13).

\section{B. Cell Density}

In earlier work by Balding and McElwain [15], Byrne and Chaplain [16] and Spill et al. [50], tipto-sprout anastomosis, and as an extension tip-totip anastomosis, were modeled via terms of the form $-\beta_{e} n e$ and $-\beta_{n} n^{2}$ (see Appendix A). In our model, tip-to-tip and tip-to-sprout anastomosis give rise to flux terms of the form $-n\left(D \nabla^{2} n-\chi \nabla \cdot(n \nabla c)\right)$ and $\left.-e\left(D \nabla^{2} n-\chi \nabla \cdot(n \nabla c)\right)\right)$, respectively, in addition to the standard sink terms $-\mu n^{2}$ and $-\mu n e$. As mentioned, anastomosis imposes occupancy restrictions on the discrete model. These effects manifest in the continuum model through the anastomosis flux terms, written as $\left(1-a_{n} n-a_{e} e\right)\left(D \nabla^{2} n-\chi \nabla \cdot(n \nabla c)\right)$ in equations $(26)$ and (31), and effectively impose density restrictions on the continuum model. If $a_{e}=0$, our model is wellposed, provided we impose initial conditions with $n \leq 1$. However, if $a_{e}=1,\left(1-a_{n} n-a_{e} e\right)$ may become negative even if the initial TC/EC densities do not exceed one. This may occur when the continuum model deviates from the discrete model due to a break-down of the independence assumption. Alternatively, when only tipto-sprout anastomosis is active $\left(a_{n}=0, a_{e}=1\right)$, it may occur due to modeling assumptions made to replicate the snail-trail process. We conclude that tip-to-sprout anastomosis should be implemented with tip-to-tip anastomosis for the model to remain well-posed when our mean-field approximation holds.

As mentioned, branching has been implemented without any volume exclusion and thus may violate cell density (occupancy) restrictions imposed by anastomosis in the continuum (discrete) model. However, provided the branching rate, $\lambda c$, is low, the model will remain wellposed.

In the next section, we will compare our onedimensional PDE model to the averaged CA simulation results to assess agreement. We will also compare Byrne and Chaplain's (BC) non-dimensional snail-trail model [16] (see Appendix A for model details) to the CA simulation results.

\section{CONTINUUM-DISCRETE COMPARISONS IN ONE DIMENSION}

\section{A. Parameter Values}

To compare discrete and continuum models, we must set and relate micro- and macro-scale parameters. There are five macro-scale parameters: $D, \chi, \lambda, \mu$ and $t_{I C}$ and six micro-scale parameters: $R, h, P_{m}, P_{p}, \tau$ and $k$. We set the macroscopic diffusion and chemotactic coefficients (using values from the BC model), $D$ and $\chi$, and $t_{I C}$, and the following micro-scale parameters in the CA model: the size of the lattice, $R$ (which determines the lattice spacing $h$ ), and the probabilities $P_{m}, P_{p}$. We then use 
the relationships in equation (24) to derive values for the micro-scale parameters $k$ and $\tau$. The value for $k$ allows us to define the remaining probabilities in our CA model, $P_{x \pm}$. From the value for $\tau$, we can then find the remaining macroscopic coefficients in our DCE-derived model, $\mu$ and $\lambda$, from equation (23). Note that we must also set $a_{n}$ and $a_{e}$ in the CA model to fix which anastomosis processes are active. These parameters, $a_{n}$ and $a_{e}$, also appear in the continuum model.

The parameter values used in the discrete and DCEderived continuum models are stated in Table I. The remaining parameter values for the $\mathrm{BC}$ model, $\lambda, \beta_{n}=$ $a_{n} \mu$ and $\beta_{e}=a_{e} \mu$ are chosen to be consistent with the values used in our continuum model.

\section{B. Continuum-Discrete Comparisons in One Dimension}

We compare the solutions (see Appendix B for numerical schemes) of the one-dimensional equations (31) and (32) and solutions to the one-dimensional BC model (equations (A1) and (A2)) to averaged CA simulation results defined in equations (12) and (13), where lattice site occupancy has been averaged over $M=200$ realizations and $R=200$ columns.

In Fig. 4 we compare solutions to our PDE model with the $\mathrm{CA}$ simulation results and solutions to the $\mathrm{BC}$ model when anastomosis is neglected, when tip-to-tip anastomosis is active and when both tip-to-tip and tip-to-sprout anastomosis are active.

We see good agreement between the CA simulation results and the DCE-derived PDEs (solutions fall within the standard deviation of the averaged CA simulation results) for both the TC and EC densities when anastomosis is neglected (Figs. 4(a), 4(b)) and when tip-to-tip anastomosis is active (Figs. 4(c), 4(d)). The agreement is poor when both tip-to-tip and tip-to-sprout anastomosis are active (Figs. 4(e), 4(f)). The removal of TCs associated with anastomosis results in a decrease in the TC density. The effect is more pronounced for tip-to-sprout anastomosis, with all TCs lost and propagation of the vascular front halted for $t \geq 0.4$.

We also compared the $\mathrm{BC}$ model to the $\mathrm{CA}$ simulation results (see Figs. 4(a)-4(f)). However, we rescaled the EC density generated by the $\mathrm{BC}$ model (equation (A2)) by 2 such that

$$
\frac{\partial e}{\partial t}=\frac{2}{h}\left|D \frac{\partial n}{\partial x}-\chi n \frac{\partial c}{\partial x}\right|
$$

In the $\mathrm{BC}$ model, the vessel/EC density is proportional to the flux of TCs in the $x$-direction. However, TCs are able to walk randomly in the $y$-direction (recall that in the CA model, a TC can move in the positive $x$-direction with probability $1 / 2$ and in the $y$-direction with probability $1 / 2$ (see Table I)). This means that TCs leave behind ECs as they move in the $y$-direction. This effect is not included in the BC model, and therefore equation (A2) underestimates the true EC density by a half. We note further that in the discrete model, if the probabilities for TC movement in the $x$ - and $y$-directions, $P_{x \pm}$ and $P_{y \pm}$, respectively, were different, then the path of the TCs would be different and the EC density would have to be rescaled as

$$
\frac{\partial e}{\partial t}=\frac{\kappa}{h}\left|D \frac{\partial n}{\partial x}-\chi n \frac{\partial c}{\partial x}\right|,
$$

where the non-negative parameter $\kappa$ accounts for EC density created via TC motion in directions other than the positive $x$-direction.

When equation (36) is used in place of equation (A2), there is good agreement between the CA simulation results and solutions to the BC model (see Figs. 4(a)-4(d)). We see poor agreement between the CA simulation results and the BC model when both tip-to-tip and tip-tosprout anastomosis are active (see Figs. 4(e), 4(f)).

The proximity of cells affects the chances of an anastomosis event, and thus, the assumption that the occupancy of lattice sites are independent may no longer hold. This is the most likely reason why our continuum model, derived using a mean-field approximation, does not capture the rapid rate of tip-to-sprout anastomosis (see Figures 4(e), 4(f)). In deriving our PDE model, we neglected terms that involve higher-order derivatives of $n$ $\left(O\left(h^{4}\right)\right.$ and higher), which describe a boundary layer in which the TC density changes rapidly. Thus, it is unlikely that these terms capture the rapid rate of tip-to-sprout anastomosis which removes all TCs and prevents propagation of the vascular front in the CA model. Including correlations in our framework would be intractable (see [47]). Therefore, we opt instead to make our CA model more biologically realistic, modifying the tip-to-sprout anastomosis process to prevent annihilation of all TCs (explained further in the next section). We then capture this more realistic behavior with our PDE model through parameter fitting.

\section{TIP-TO-SPROUT ANASTOMOSIS: EXCLUDING SELF-LOOPS}

In our CA model, tip-to-sprout anastomosis occurs when a TC encounters an EC. Extensive simulations (results not shown) reveal that eventually all TCs are annihilated even if they are initially well separated. We deduce that diffusive motion of the TCs in the $y$-direction and, in general, in directions other than the positive $x$ direction, causes TCs to anastomose with ECs in their own sprout (self-loops), creating a stunted sprout. All TCs are annihilated through these self-loops. Such selfloops are inefficient as they will not become perfused or connect the tumor to the limbus (blood supply). Further, in practice, it is unlikely that many stunted sprouts occur, and if they do, most will likely regress [5].

To make our discrete model more biologically realistic, we modify our CA model to preclude self-loops during 
TABLE I. CA and DCE-derived PDE model parameters

\begin{tabular}{cccccccccccccccc}
\hline \hline$R$ & $P_{m}$ & $P_{p}$ & $h$ & $\tau$ & $k$ & $g_{x}$ & $g_{y}$ & $P_{x+}$ & $P_{x-}$ & $P_{y \pm}$ & $D$ & $\chi$ & $\lambda$ & $\mu$ & $t_{I C}$ \\
\hline 200 & 1 & $10^{-3}$ & $\frac{1}{200}$ & $\frac{1}{160}$ & 100 & 1 & 0 & $\frac{1}{2}$ & 0 & $\frac{1}{4}$ & $10^{-3}$ & 0.4 & 0.16 & 160 & 0.2 \\
\hline \hline
\end{tabular}

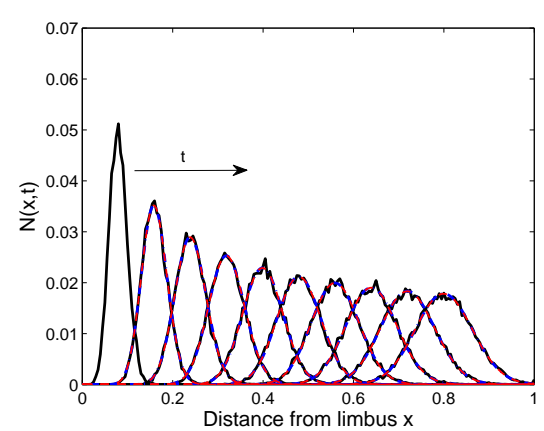

(a) Tip cell density (no anastomosis)

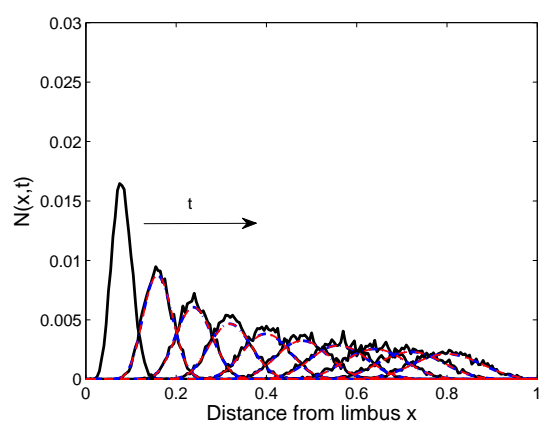

(c) Tip cell density (tip-to-tip anastomosis)

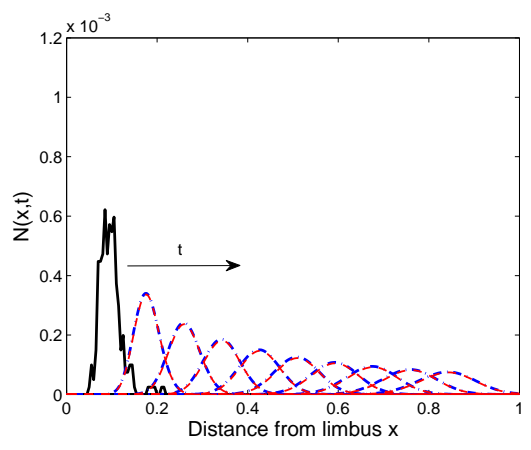

(e) Tip cell density (tip-to-tip and tip-to-sprout anastomosis)

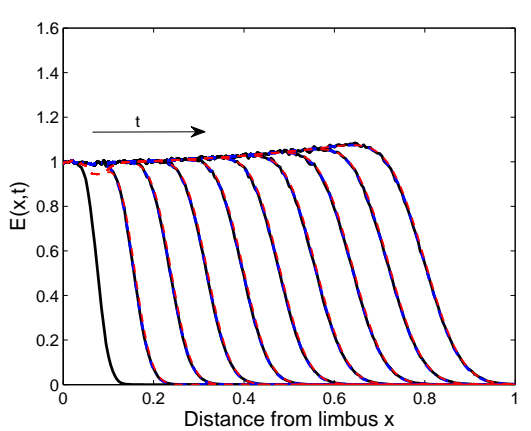

(b) Endothelial cell density (no anastomosis)

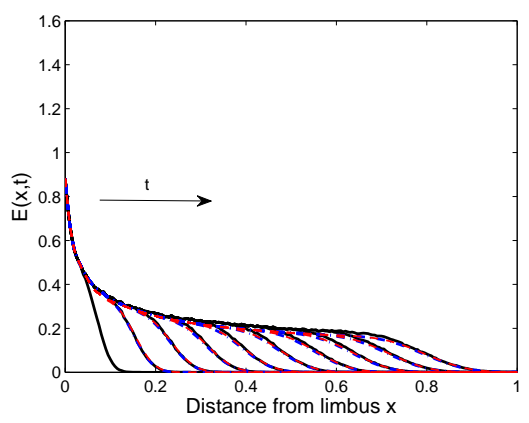

(d) Endothelial cell density (tip-to-tip anastomosis)

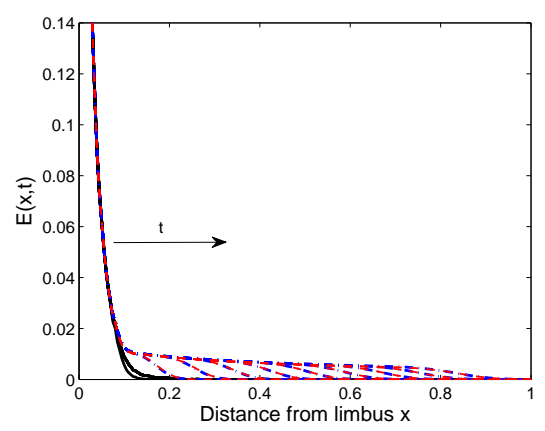

(f) Endothelial cell density (tip-to-tip and tip-to-sprout anastomosis)

FIG. 4. (Color online) (a), (b) Tip cells (TCs) migrate via diffusion and chemotaxis towards the TAF source at $x=1$, leaving behind a trail of endothelial cells (ECs). (c), (d) Tip-to-tip anastomosis annihilates TCs as they migrate towards the TAF source. (e), (f) Tip-to-sprout anastomosis annihilates all TCs such that the vascular front ceases to propagate towards the TAF source. Column-averaged CA simulation results (black solid curve) are shown from $t=0.2$ to $t=2$ in 0.2 intervals, incorporating TC movement, branching, EC creation, (c), (d) tip-to-tip anastomosis and (e), (f) tip-to-tip and tip-to-sprout anastomosis. The CA simulation results at $t_{I C}=0.2$ are used as the initial conditions in the DCE-derived PDE model (blue (dark gray) dashed-dot curve), equations (31) and (32), and the BC model (red (light gray) dashed curve), equations (A1) and (36), with the PDE solutions shown from $t=0.4$ to $t=2$. Discrete and PDE model parameters are given in Table I with the parameters controlling anastomosis specified in the DCE-derived PDE model as (a), (b) $a_{n}=a_{e}=0,(\mathrm{c}),(\mathrm{d}) a_{n}=1, a_{e}=0$, (e), (f) $a_{n}=a_{e}=1$. The corresponding anastomosis parameters for the BC model can be calculated as $\beta_{n}=160 a_{n}$ and $\beta_{e}=160 a_{e}$. The arrow shows the direction of increasing time. 
tip-to-sprout anastomosis in the following way. When a TC attempts to move to a site occupied by an EC (identified as the last EC to be created at that site), the move is aborted if that EC belongs to the same sprout as the TC. If the EC belongs to a different sprout, then anastomosis occurs.

We must account for this modification to the CA model in our PDE model. As it would be intractable to keep track of ECs in a particular sprout in our DCEframework, we instead allow the parameter that controls the rate of tip-to-sprout anastomosis, $a_{e}$, to vary and estimate it by fitting PDE solutions to the CA simulations results with self-loops excluded.

\section{A. Parameter Fitting}

When we fit our PDE model to the averaged CA simulation results, we fix $a_{n}=1$ and suppose that $a_{e}$ is no longer a binary variable. With $0<a_{e} \leq 1$, we can control the rate of tip-to-sprout anastomosis. In the same way, when fitting the $\mathrm{BC}$ model, we fix $\beta_{n}=160$ and suppose $0<\beta_{e} \leq 160$ (see Appendix B for details).

The resulting parameter estimates and the $95 \%$ confidence intervals are stated in Table II.

TABLE II. Least squares fit: tip-to-sprout anastomosis parameters

\begin{tabular}{ccc}
\hline \hline Parameter (Model) & Value & $95 \%$ Confidence Interval \\
\hline$a_{e}$ (DCE-derived) & 0.0343 & \pm 0.0003 \\
$\beta_{e}$ (BC Model) & 5.0648 & \pm 0.0423 \\
\hline \hline
\end{tabular}

\section{B. Tip-to-Sprout Anastomosis:}

Continuum-Discrete Comparisons in One-Dimension

With $a_{e}=0.0343$ in equations (31) and (32) (and $\beta_{e}=5.0648$ in equations (A1) and (36)), there is good agreement between the PDE models and the CA simulation results (see Fig. 5). Furthermore, when self-loops are excluded, the vascular front continues to propagate towards the TAF source as the TCs are not exhausted (see Figs. 4(e), 4(f)).

\section{DISCUSSIONS AND CONCLUSIONS}

Directly relating discrete and continuum models, and therefore micro- and macro-scales, allows not only for a greater understanding of angiogenesis but also model validation at both scales. We have developed a twodimensional CA model of angiogenesis in the corneal assay, based on the snail-trail process. Using a meanfield approximation and discrete conservation equations

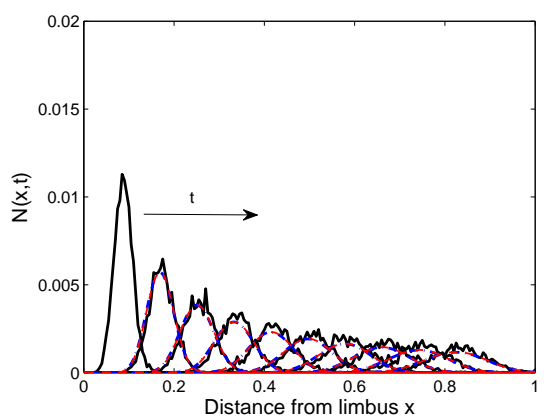

(a) Tip cell density

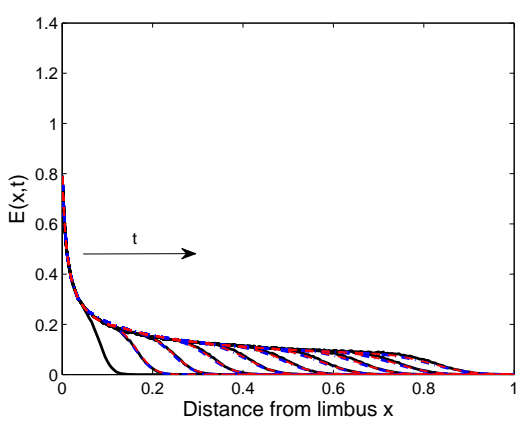

(b) Endothelial cell density

FIG. 5. (Color online) Tip-to-sprout anastomosis decreases tip cell (TC) and endothelial cell (EC) densities. Columnaveraged CA simulation results (black solid curve) are shown from time $t=0.2$ to 2 in increments of 0.2 , incorporating TC movement, branching, tip-to-tip, tip-to-sprout anastomosis excluding self-loops and EC creation. Solutions to the DCE-derived PDE model (blue (dark gray) dashed-dot curve), equations (31) and (32), and the BC model (red (light gray) dashed curve), equations (A1) and (36), are shown from $t=0.4$ to $t=2$ with the averaged CA simulation results at $t_{I C}=0.2$ used as the initial conditions. Discrete and PDE model parameters are given in Table I with the parameters controlling tip-to-sprout anastomosis specified as $a_{e}=0.0343$ and $\beta_{e}=5.0648$ in the DCE-derived model and the BC model, respectively. The parameters $a_{e}$ and $\beta_{e}$ have been found by fitting the PDE solutions to the CA simulation results. The arrow shows the direction of increasing time.

(DCEs), we have derived a new continuum model of angiogenesis consisting of a set of PDEs for the spatiotemporal evolution of the tip cell (TC) and endothelial cell (EC) densities. From this PDE model, we derived a one-dimensional continuum model that represents twodimensional angiogenesis in the corneal assay.

We found that EC creation due to TC movement can be modeled as a source term of TCs on the macro-scale. In contrast, phenomenological models $[15,16]$ assume that EC creation is proportional to the flux of TCs in the direction of increasing TAF concentration (i.e. towards the TAF source). We have found that these snailtrail $[15,16]$ models qualitatively account for vessel formation (EC creation) in two dimensions. However, for these models to accurately account for vessel formation 
in two dimensions, the PDEs for EC density must be scaled by a factor to account for TC motility in directions other than towards the TAF source. This scaling factor and, therefore vessel formation, depends on the micro-scale behavior of TCs.

Additionally, we found that anastomosis imposes cell occupancy and density restrictions on the discrete model and continuum model, respectively. As a result, for our continuum model to remain well-posed, tip-to-sprout anastomosis should be implemented when tip-to-tip anastomosis is active. The density restrictions are as follows: when tip-to-tip anastomosis is active, the TC density should not exceed one, and when both tip-to-tip and tip-to-sprout anastomosis are active, the combined cell density (TC and EC) should not exceed one. These findings differ from existing continuum models [15, 16, 50], which model anastomosis entirely through phenomenological sink terms, which do not impose any restrictions on cell density. Branching was implemented as a separate process in our model, and thus may not adhere to the density restrictions imposed by anastomosis. However, provided the branching rate is low, the continuum model will remain well-posed.

When both tip-to-tip and tip-to-sprout anastomosis are active, we found that our PDE model does not agree with the CA model. In particular, our PDE model does not capture the rapid rate of tip-to-sprout anastomosis which annihilates all TCs in the CA model. This TC exhaustion occurs as a result of self-loops in the CA model. We postulate that the discrepancy between the discrete and continuum model arises due to a break-down of the independence assumption (mean-field approximation) introduced by tip-to-sprout anastomosis. In order to produce a more realistic discrete model of angiogenesis (a TC front which propagates towards the TAF source), we modified our CA model to prevent the formation of selfloops during tip-to-sprout anastomosis. As it would have been intractable to use our DCE-framework to account for self-loops, we fitted the parameter in the continuum model that controls the rate of tip-to-sprout anastomosis, $a_{e}$, to the averaged CA simulation results with self-loops excluded.

Our PDE models were derived by assuming that the lattice spacing $h$ is small $(h \ll 1)$ and terms of $O\left(h^{4}\right)$ and higher were negligible. In addition, for our model to be valid, the assumption that the occupancy of lattice sites are independent must hold. This places constraints on the probabilities for movement, $P_{m}$, and branching (TC proliferation), $P_{p}$, in the CA model. Anastomosis may also lead to a break-down of the independence assumption. However, we have shown that we can account for the complexity introduced by anastomosis by fitting the relevant parameters in the PDE model to the $\mathrm{CA}$ simulation results. We also remark that we could relax the assumptions on the TAF field, and develop a CA and corresponding one- and two-dimensional continuum models for the TC and EC densities for a two-dimensional and/or time-dependent TAF field. In future, we will ex- tend this work to comprehensively understand the effects of volume exclusion, and determine how predictions differ across different continuum models of angiogenesis.

\section{ACKNOWLEDGMENTS}

S. Pillay acknowledges funding from the Natural Sciences and Engineering Research Council of Canada (Postgraduate Scholarship), and the Schlumberger Foundation (Faculty for the Future Fellowship).

\section{Appendix A: Review of Byrne and Chaplain's Snail-Trail Model [16]}

We use the following modified version of Byrne and Chaplain's non-dimensional model [16]

$$
\begin{aligned}
& \frac{\partial N}{\partial t}=D \frac{\partial^{2} N}{\partial x^{2}}-\chi \frac{\partial}{\partial x}\left(N \frac{\partial c}{\partial x}\right)+\lambda N c-\beta_{e} N E-\beta_{n} N^{2}, \\
& \frac{\partial E}{\partial t}=\frac{1}{h}\left|D \frac{\partial N}{\partial x}-\chi N \frac{\partial c}{\partial x}\right|,
\end{aligned}
$$

where $N(x, t)$ denotes TC density in units of number of TCs per unit area, EC density, $E(x, t)$, in units of of number of ECs per unit area and TAF concentration (molar concentration) per unit area, $c(x, t)$. The model is defined on $(x, t) \in[0,1] \times[0, \infty)$ where the TAF source is located at $x=0$ and the limbus at $x=1$, subject to no flux boundary conditions (equation (33)), with initial conditions and linear TAF profile taken from the CA model. In this model, TCs migrate via diffusion and chemotaxis, new TCs emerge from existing TCs at a rate $\lambda c$, tip-to-sprout anastomosis is modeled through the sink term $-\beta_{e} N E$, and ECs form in response to TC flux. We have introduced tip-to-tip anastomosis in the $\mathrm{BC}$ model by including a term of the form $-\beta_{n} n^{2}$ in equation (A1).

The original $\mathrm{BC}$ model represented the network in terms of vessel length per unit area, $\rho$ (i.e. not EC density). To relate the vessel density to EC density extracted from the CA model, measured in units of number of ECs averaged over the $y$-direction (i.e per unit area), we converted vessel density from a length to a number density. The length of a cell is defined by the lattice spacing in our model, $h$. Thus, to convert vessel density to EC density, we must divide $\rho$ by $h$ such that

$$
E=\frac{\rho}{h} .
$$

As in Spill et al. [50], we have used the absolute value of the TC flux in equation (A2) to guarantee that the source of ECs generated by the TC flux is non-negative. We have also neglected branching from ECs and vessel regression, originally included in the BC model. Following [16], we set $D=10^{-3}$ and $\chi=0.4$, with $\lambda, \beta_{e}$ and $\beta_{n}$ chosen to be consistent with our continuum model (see 
Table I). With these values for $D$ and $\chi$, the TC flux is always negative and thus the absolute value in equation (A2) can be replaced by a negative sign.

\section{Appendix B: Numerical Schemes}

\section{CA Algorithm}

The algorithm for the CA model follows:

While $\bar{K} \leq \bar{K}_{\text {final }}$ and TCs have not reached $i=R$

1. Choose $\bar{N}$ TCs at random with replacement

Loop 1: For 1 to $\bar{N}$

(a) Choose a random number, $\bar{T} \in[0,1]$

(b) If $\bar{T} \leq P_{m}$, then the TC to move as follows:

- A random number $\bar{S} \in[0,1]$ is chosen

- The TC moves according to probabilities $P_{x \pm}, P_{y \pm}$ and an EC is left behind

- If $a_{n}=1$ and the target site is occupied by TCs, tip-to-tip anastomosis occurs

- Otherwise if $a_{e}=1$, and the target site is occupied by ECs, tip-to-sprout anastomosis occurs

- Otherwise the TC remains at the target site

End Loop 1

2. Choose $\bar{N}$ TCs at random with replacement

Loop 2: For 1 to $\bar{N}$

(a) Choose a random number, $\bar{R} \in[0,1]$

(b) If $\bar{R} \leq P_{b}=P_{p} c$, then branching occurs

End Loop 2

3. Increment time step: $\bar{K}=\bar{K}+1$

End While Loop

\section{PDE Solvers}

Equations (31), (32), (A1), (A2) and (37) are solved numerically using the method of lines with a finite difference scheme in space (upwinding for equations (A2) and (37)). The resulting ordinary differential equations in time are solved over $t \in[0.2,2]$ using MATLAB's ode15s solver, which implements adaptive time-stepping [54].

\section{Parameter Fitting}

We carry out the parameter fitting using a non-linear least squares fitting procedure using MATLAB's lsqnon- lin routine, which implements a trust-region-reflective algorithm [55, 56], with the PDEs solved as described above. We fit the parameters using averaged CA simulation results over $t \in[0.2,2]$ in intervals of 0.2 for both the TC and EC densities.

[1] D. Hanahan and R. A. Weinberg, Cell 100, 57 (2000).

[2] D. Hanahan and R. A. Weinberg, Cell 144, 646 (2011). 
[3] M. Klagsbrun and M. A. Moses, Chemistry \& Biology 6, R217 (1999).

[4] J. Folkman and M. Klagsbrun, Science 235, 442 (1987).

[5] P. Carmeliet and R. K. Jain, Nature 473, 298 (2011).

[6] M. Potente, H. Gerhardt, and P. Carmeliet, Cell 146, 873 (2011).

[7] D. H. Ausprunk and J. Folkman, Microvascular Research 14, 53 (1977).

[8] P. Benitez and S. Heilshorn, Mechanical and Chemical Signaling in Angiogenesis, edited by C. A. Reinhart-King (Springer-Verlag, 2013) Chap. Microfluidic Devices for Quantifying the Role of Soluble Gradients in Early Angiogenesis, pp. 47-60.

[9] V. R. Muthukkaruppan, L. Kubai, and R. Auerbach, Journal of the National Cancer Institute 69, 699 (1982).

[10] R. K. Jain, Nature Medicine 7, 987 (2001).

[11] R. S. Samant and L. A. Shevde, Oncotarget 2, 122 (2011).

[12] P. Carmeliet, Nature 438, 932 (2005).

[13] N. V. Mantzaris, S. Webb, and H. G. Othmer, Journal of Mathematical Biology 49, 111 (2004).

[14] M. Scianna, C. G. Bell, and L. Preziosi, Journal of Theoretical Biology 333, 174 (2013).

[15] D. Balding and D. L. S. McElwain, Journal of Theoretical Biology 114, 53 (1985).

[16] H. M. Byrne and M. A. J. Chaplain, Bulletin of Mathematical Biology 57, 461 (1995).

[17] R. Auerbach, R. Lewis, B. Shinners, L. Kubai, and N. Akhtar, Clinical Chemistry 49, 32 (2003).

[18] M. A. Gimbrone, R. S. Cotran, S. B. Leapman, and J. Folkman, Journal of the National Cancer Institute 52, 413 (1974).

[19] D. Schulz, M. E. Iliev, B. E. Frueh, and D. Goldblum, Vision Research 43, 723 (2003).

[20] H. Zhang, L. Wang, Y. Xie, S. Liu, and X. D. et al., Experimental Eye Research 115, 255 (2013).

[21] M. A. J. Chaplain and M. Stuart, IMA Journal of Mathematics Applied in Medicine \& Biology 10, 149 (1993).

[22] M. A. J. Chaplain, Acta Biotheoretica 43, 387 (1995).

[23] M. A. J. Chaplain, Mathematical and Computer Modelling 23, 47 (1996).

[24] A. J. Connor, R. P. Nowak, E. Lorenzon, M. Thomas, and F. H. et al., Journal of the Royal Society Interface 12, 20150546 (2015).

[25] C. L. Stokes and D. A. Lauffenburger, Journal of Theoretical Biology 152, 377 (1991).

[26] M. J. Plank and B. D. Sleeman, Bulletin of Mathematical Biology 66, 1785 (2004).

[27] A. R. A. Anderson and M. A. J. Chaplain, Bulletin of Mathematical Biology 60, 857 (1998).

[28] M. A. J. Chaplain, Journal of Neuro-Oncology 50, 37 (2000).

[29] K. Bentley, G. Mariggi, H. Gerhardt, and P. A. Bates, PLoS Computational Biology 5, e1000549 (2009).

[30] K. Bentley, H. Gerhardt, and P. A. Bates, Journal of Theoretical Biology 250, 25 (2008).
[31] H. A. Harrington, M. Maier, L. Naidoo, N. Whitaker, and P. Kevrekidis, Mathematical and Computer Modelling , 513 (2007).

[32] S. Tong and F. Yuan, Microvascular Research , 14 (2001).

[33] S. Tong and F. Yuan, Microvascular Research 75, 16 (2008).

[34] G. Vilanova, I. Colominas, and H. Gomez, Computational Mechanics 53, 449 (2014).

[35] A. Stéphanou, S. R. McDougall, A. R. A. Anderson, and M. A. J. Chaplain, Mathematical and Computer Modelling 41, 1137 (2005).

[36] M. R. Owen, T. Alarcón, P. K. Maini, and H. M. Byrne, Journal of Mathematical Biology 58, 689 (2009).

[37] H. Perfahl, H. M. Byrne, T. Chen, V. Estrella, and T. A. et al., PLoS One 6, e14790 (2011).

[38] R. E. Baker, C. A. Yates, and R. Erban, Bulletin of Mathematical Biology 72, 719 (2010).

[39] M. J. Simpson, K. A. Landman, and B. D. Hughes, Physica A 389, 3779 (2010).

[40] M. J. Simpson, K. A. Landman, and B. D. Hughes, Physica A 388, 399 (2009).

[41] M. J. Simpson, K. A. Landman, B. D. Hughes, and A. E. Fernando, Physica A 389, 1412 (2010).

[42] C. J. Penington, B. D. Hughes, and K. A. Landman, Physical Review E 84, 041120 (2011).

[43] R. J. H. Ross, C. A. Yates, and R. E. Baker, Mathematical Biosciences 264, 108 (2015).

[44] J. D. Hywood, E. J. Hackett-Jones, and K. A. Landman, Physcial Review E 88, 032704 (2013).

[45] K. J. Davies, J. E. F. Green, N. G. Bean, B. J. Binder, and J. V. Ross, Mathematical Biosciences 253, 63 (2014).

[46] E. A. Codling, M. J. Plank, and S. Benhamou, Journal of the Royal Society Interface 5, 813 (2008).

[47] R. E. Baker and M. J. Simpson, Physical Review E 82, 041905 (2010).

[48] D. C. Markham, R. E. Baker, and P. K. Maini, Discrete and Continuous Dynamical Systems 34, 5123 (2014).

[49] D. C. Markham, Spatial Correlation Models for Cell Populations, D.Phil thesis, University of Oxford (2014).

[50] F. Spill, P. Guerrero, T. Alarcón, P. K. Maini, and H. M. Byrne, Journal of Mathematical Biology 70, 485 (2015).

[51] L. L. Bonilla, V. Capasso, M. Alvaro, and M. Carretero, Physical Review E 90, 062716 (2014).

[52] M. E. Orme and M. A. J. Chaplain, IMA Journal of Mathematics Applied in Medicine \& Biology 14, 189 (1997).

[53] R. Blanco and H. Gerhardt, Cold Spring Harbor Perspectives in Medicine 3, a006569 (2013).

[54] L. F. Shampine and M. W. Reichelt, SIAM Journal on Scientific Computing 18, 1 (1997).

[55] T. F. Coleman and Y. Li, SIAM Journal on Optimization 6, 418 (1996).

[56] T. F. Coleman and Y. Li, Mathematical Programming 67, 189 (1994). 